Research Paper

\title{
Evaluation and validation of the diagnostic value of the apparent diffusion coefficient for differentiating early-stage endometrial carcinomas from benign mimickers at 3T MRI
}

\author{
Xue Wang ${ }^{1, *}$, Yu Zhao ${ }^{2, *}$, Yumin Hu${ }^{1}$, Yongjin Zhou ${ }^{1}$, Xinjian Ye ${ }^{1}$, Kun Liu ${ }^{1}$, Guanghui \\ Bai $^{1}$, Anna Guo ${ }^{1}$, Meimei Du${ }^{1}$, Lezhen Jiang ${ }^{1}$, Jinhong Wang ${ }^{3, *}$ and Zhihan Yan ${ }^{1, *}$ \\ ${ }^{1}$ Department of Radiology, The Second Affiliated Hospital and Yuying Children's Hospital of Wenzhou Medical University, \\ Wenzhou 325027, China \\ ${ }^{2}$ Department of Gynecology and Obstetrics, The Second Affiliated Hospital and Yuying Children's Hospital of Wenzhou \\ Medical University, Wenzhou 325027, China \\ ${ }^{3}$ Department of Medical Imaging, Shanghai Mental Health Center, Shanghai Jiao Tong University School of Medicine, Shanghai \\ 200030, China \\ *These authors contributed equally to this work
}

Correspondence to: Zhihan Yan, email: yanzhihanwz@163.com

Jinhong Wang, email: jinhongw2004@foxmail.com

Keywords: magnetic resonance imaging, endometrial lesions, diffusion-weighted imaging, apparent diffusion coefficient, diagnostic accuracy

Received: January 16, 2017 Accepted: June 04, $2017 \quad$ Published: June 16, 2017

Copyright: Wang et al. This is an open-access article distributed under the terms of the Creative Commons Attribution License 3.0 (CC BY 3.0 ), which permits unrestricted use, distribution, and reproduction in any medium, provided the original author and source are credited.

\section{ABSTRACT}

Previous researchers obtained various apparent diffusion coefficient (ADC) cutoff values to differentiate endometrial carcinoma from benign mimickers with 1.5T magnetic resonance imaging (MRI). Few studies have used 3T MRI or validated the effectiveness of these cutoff ADC values prospectively. This study was designed in two stages to obtain a cutoff ADC value at $3 T$ MRI and to validate prospectively the role of the ADC value. First, we conducted a retrospective study of 60 patients to evaluate the diagnostic value of ADC by obtain a theoretical cutoff ADC value for differentiating between benign and malignant endometrial lesions. Student's t test revealed that ADC values for stage I endometrial carcinomas were significantly lower than those for benign lesions. The area under the curve value of the receiver operating characteristic curve was 0.993 , and the cutoff ADC value was $0.98 \times 10^{-3} \mathrm{~mm}^{2} / \mathrm{s}$. The sensitivity, specificity, and overall accuracy of diagnosing stage I endometrial carcinoma were $100 \%, 97.1 \%$, and $98.3 \%$, respectively. Second, we conducted a prospective study of $\mathbf{2 6}$ patients to validate the use of the cutoff ADC value obtained in the study's first stage. The sensitivity, specificity, and overall accuracy for differentiating malignant from benign endometrial lesions based on the cutoff ADC value obtained earlier were as follows: radiologist 1 attained $86.67 \%, 100.0 \%$, and $92.31 \%$, respectively; radiologist 2 attained $86.67 \%, 91.0 \%$, and $88.5 \%$, respectively. Our results suggest that ADC values could be a potential biomarker for use as a quantitative and qualitative tool for differentiating between early-stage endometrial carcinomas and benign mimickers.

\section{INTRODUCTION}

Endometrial cytology, biopsy, and curettage have been the mainstays for accurately diagnosing lesions in the endometrial cavity. As these procedures are commonly performed in a blind manner, however, they do not always provide a definitive diagnosis. In addition, they are difficult to perform in patients with vaginal or cervical stenosis [1-3]. Transvaginal sonography has been proposed as the first-line diagnostic tool for evaluating endometrial 
thickness, but operator experience, the position of the uterus, and vaginal anomalies limit its usefulness. Because of its excellent soft tissue contrast resolution, multi-planar imaging capability, and post-processing tools, magnetic resonance imaging (MRI) is increasingly being applied for diagnosing endometrial diseases and as a problemsolving tool when there is a diagnostic dilemma [4-6].

Clinically, however, using conventional MRI to differentiate endometrial carcinoma from benign mimickers, such as polyps and submucosal myomas, remains challenging [7]. In contrast, diffusion-weighted imaging (DWI) is based on diffused, random motion of water molecules and provides information about cell density of a given tissue and the integrity of cell membranes [8]. It can be quantified using an apparent diffusion coefficient (ADC) or qualitatively assessed in biological tissues [9, 10].

ADC values have been shown to be decreased in various tumors [11-16]. The assessment of endometrial carcinomas versus benign lesions in the endometrial cavity using DWI and 1.5T MRI has been reported [14, 17-19]. As $3 \mathrm{~T}$ MRI scanners are increasingly used in clinical practice, it is necessary to investigate the cutoff ADC value for differentiating endometrial carcinomas from benign mimickers at 3T MRI. To our knowledge, there are few data prospectively validating the diagnostic performance of the cutoff ADC value for lesions in the endometrial cavity using 3T MRI.

In this study, we first obtained an ADC cutoff value via a retrospective study at $3 \mathrm{~T}$ MRI. The ADC value was then validated prospectively to differentiate stage I endometrial carcinoma and its benign mimickers. This study aimed to investigate the feasibility of using the ADC value to differentiate early-stage endometrial carcinomas from benign mimickers.

\section{RESULTS}

For the first stage of the study (May 2012 to September 2014), we enrolled 60 patients (ages 4575 years, mean 58 years) with lesions in the uterine endometrial cavity. The histological types and frequencies of the lesions were as follows: stage Ia endometrial carcinomas $(n=22)$; stage $\mathrm{Ib}$ endometrial carcinomas $(n=3)$; endometrial polyps $(n=15)$; submucosal leiomyomas $(n=20)$. These lesions appeared as isointense to slightly high signal intensity compared with the normal myometrium on T2-weighted imaging (T2WI). In all, 35 benign endometrial lesions presented with slightly low or intermediate signal intensity on T2WI. Among 25 endometrial cancers, 11 had intratumoral cystic changes and 3 had fibrous cores, whereas among the 35 benign lesions, only 3 showed intratumoral cystic changes and 3 had fibrous cores. T2WI revealed four pedunculated submucosal leiomyomas. Thus, the presence of these three features (signal intensity, cystic changes, fibrous cores) was not significantly different between endometrial carcinomas and benign uterine lesions $(P=0.079)$. When combining the results of T2WI and DWI, however, myometrial invasion was seen in 22 of the 25 endometrial carcinomas. No myometrial invasion was found in the benign lesions (Figure 1). All 25 stage I endometrial carcinomas showed
A

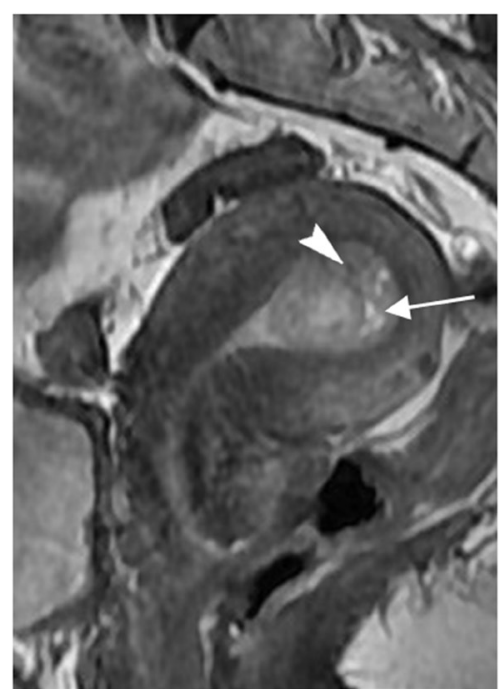

B

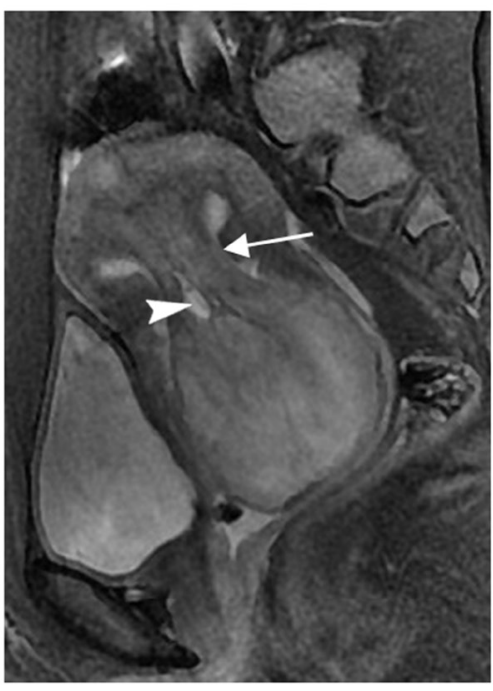

C

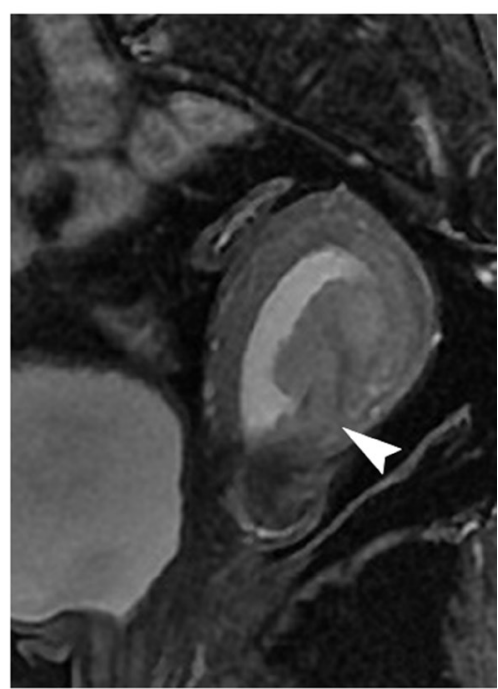

Figure 1: Three women with histopathologically proven uterine polyps, submucosal myoma, and stage I endometrial carcinoma, respectively, on sagittal T2-weighted imaging (T2WI). (A) Polyp shows slightly heterogeneous hyperintensity, with fibrous cores (arrow) and small, high-intensity intratumoral cysts (arrowhead). (B) Submucosal myoma shows slight hyperintensity compared with the normal outer myometrium. A peduncle (arrow) from the uterine fundus and intratumoral cysts (arrowhead) are seen. (C) Intensity of a stage $\mathrm{Ib}$ endometrial carcinoma was equal to that of the normal outer myometrium. Local myometrium was not consistent (arrowhead). 
high signal intensity on DWI as well as hypointensity $(25 / 25)$ and blue foci $(25 / 25)$ on the ADC map. All 35 benign endometrial lesions showed slight hypointensity or hyperintensity on DWI as well as hypointensity (35/35) and green foci $(30 / 35)$ on the ADC map. Some blue foci were seen in one polyp and four submucosal myomas (Figure 2). The ADC value $\left(\times 10^{-3} \mathrm{~mm}^{2} / \mathrm{s}\right)$ of stage I endometrial carcinomas $(0.80 \pm 0.10)$ was significantly lower than that of the benign endometrial lesions (1.43 \pm $0.25)(P=0)$. The mean ADC value for the endometrial polyps $(1.53 \pm 0.23)$ was significantly higher than that for the submucosal leiomyomas $(1.35 \pm 0.25)(P=0.032)$. The area under the receiver operating characteristic (ROC) curve was 0.993 . The cutoff ADC value obtained was $0.98 \times 10^{-3} \mathrm{~mm}^{2} / \mathrm{s}$ for achieving the highest accuracy in differentiating stage I endometrial carcinomas from benign endometrial lesions (Figure 3). The sensitivity, specificity, and overall accuracy for diagnosing of stage I endometrial carcinomas were $100 \%(25 / 25), 97.1 \%(34 / 35)$, and $98.3 \%$ (59/60), respectively, whereas the accuracy range based on the various cutoff ADC values in previous studies was $83.3 \%-93.3 \%$ (Table 1).
For the second stage of the study (October 2014 to November 2015), the study group included 26 patients (ages 30-70 years, mean 60 years) with lesions in the uterine endometrial cavity. The histological types and frequencies of the lesions were as follows: stage Ia endometrial carcinoma $(n=9)$; stage $\mathrm{Ib}$ endometrial carcinoma $(n=1)$; stage IIa endometrial carcinomas $(n=5)$; endometrial polyps $(n=4)$; submucosal leiomyomas $(n=7)$. The kappa value for agreement of the measured ADC values between two radiologists was 0.846 $(>0.75)$. The sensitivity, specificity, and overall accuracy of radiologist 1 (based on the cutoff ADC value obtained in stage 1 of the study) were $86.67 \%(13 / 15), 100.0 \%$ $(11 / 11)$, and $92.31 \%(24 / 26)$, respectively, and those of radiologist 2 were $86.67 \%(13 / 15), 91.0 \%(10 / 11)$, and $88.5 \%(23 / 26)$, respectively.

\section{DISCUSSION}

Previous researchers $[14,15,17,19,20]$ tried to find a simple, accurate way to differentiate between benign and malignant endometrial lesions. They used various cutoff
A

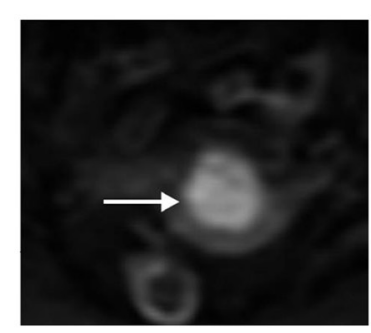

D

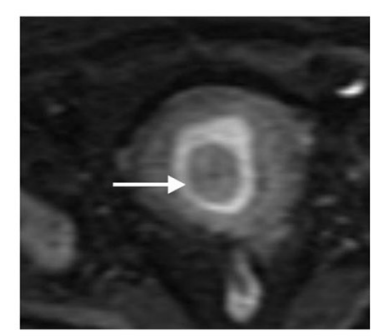

G

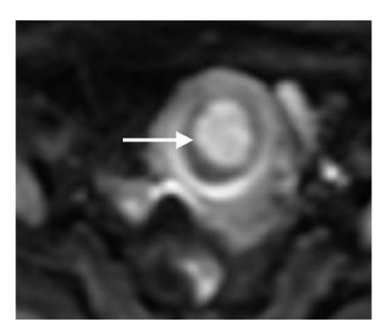

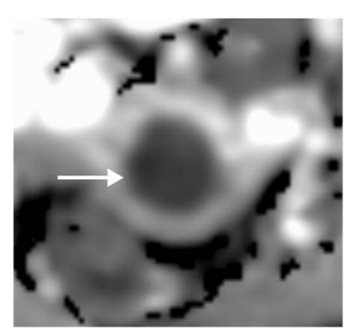

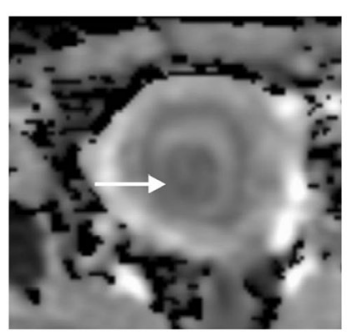

H

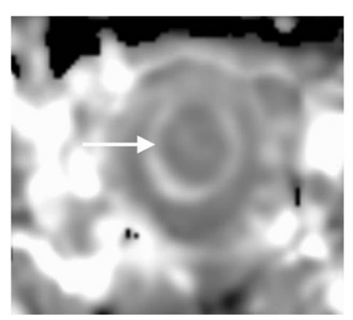

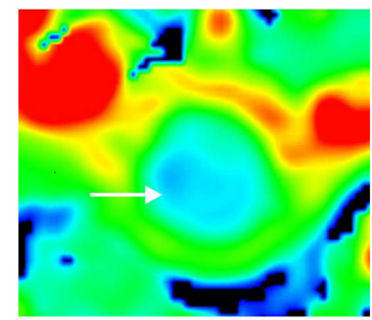

F

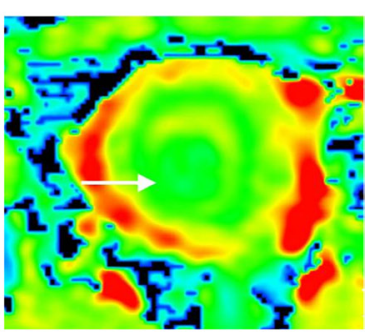

I

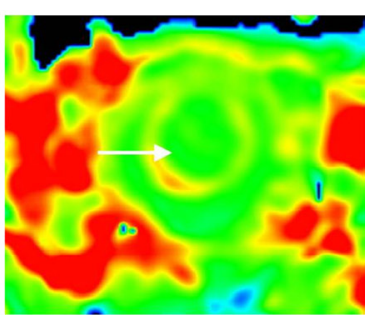

Figure 2: Three women with histopathologically proven stage I endometrial carcinoma, endometrial polyp, and a submucosal myoma, respectively, on axial diffusion-weighted imaging (DWI) and apparent diffusion coefficient (ADC) maps. (A-C) Stage I endometrial carcinoma shows hyperintensity on DWI (arrow), hypointensity, and blue foci on the ADC map (arrow). (D-F) Endometrial polyp shows hypointensity on DWI (arrow), light hypointensity, and blue foci on the ADC map (arrow). (G-I) Submucosal myoma shows light hyperintensity on DWI (arrow), light hypointensity, and green foci on the ADC map (arrow). 
Table 1: ADC values from the literature and this study

\begin{tabular}{|c|c|c|c|c|c|c|}
\hline Authors & $\begin{array}{l}\text { Magnetic field } \\
\text { strength }\end{array}$ & $\begin{array}{c}\text { b-values }\left(\mathrm{s} / \mathrm{mm}^{2}\right) \\
\text { applied }\end{array}$ & $\begin{array}{c}\text { Suggested cutoff } \\
\text { ADC value } \\
\left(\times 10^{-3} \mathrm{~mm}^{2} / \mathrm{s}\right)\end{array}$ & $\begin{array}{c}\text { Sensitivity } \\
(\%)\end{array}$ & $\begin{array}{l}\text { Specificity } \\
(\%)\end{array}$ & $\begin{array}{c}\text { Accuracy } \\
(\%)\end{array}$ \\
\hline Kilickesmez et al. [15] & $1.5 \mathrm{~T}$ & $0,500,1000$ & 1.05 & 100 & 88.6 & 93.3 \\
\hline Fujii et al. [14] & $1.5 \mathrm{~T}$ & 0,1000 & 1.15 & 100 & 85.7 & 91.7 \\
\hline Takeuchi et al. [17] & $1.5 \mathrm{~T}$ and $3 \mathrm{~T}$ & 0,800 & 1.2 & 100 & 82.9 & 90.0 \\
\hline Karakas et al. [20] & $1.5 \mathrm{~T}$ & $0,500,1000$ & 0.908 & 88 & 97.1 & 93.3 \\
\hline Bharwani et al. [19] & $1.5 \mathrm{~T}$ & $0,50,100,250,500,750$ & 1.28 & 100 & 71.4 & 83.3 \\
\hline This study & $3 \mathrm{~T}$ & 0,1000 & 0.98 & 100 & 97.1 & 98.3 \\
\hline
\end{tabular}

ADC: apparent diffusion coefficient.

ADC values as imaging biomarkers to achieve this goal. To our knowledge, however, few studies used 3T MRI, and few validated the effectiveness of these cutoff ADC values. The present study was performed in two stages to, first, obtain a cutoff ADC value at $3 \mathrm{~T}$ MRI and, second, prospectively validate use of the ADC value.

During the first stage, we obtained a theoretical cutoff ADC value of $0.98 \times 10^{-3} \mathrm{~mm}^{2} / \mathrm{s}$ for differentiation between benign and malignant endometrial lesions at $3 \mathrm{~T}$ MRI. Using the cutoff ADC value during the second stage of the study, we prospectively assessed its sensitivity, specificity, and accuracy for distinguishing malignant endometrial lesions from benign lesions. We found that the sensitivity, specificity, and accuracy for diagnosing endometrial carcinomas were high, indicating that the cutoff ADC value could be used as an imaging biomarker to differentiate between benign and malignant endometrial lesions.

Conventional MRI sequences, such as T1WI and T2WI, were generally use for evaluating endometrial lesions. Park et al. [7] reported that, on T2WI, intratumoral cystic changes and fibrous cores were seen less frequently in stage I endometrial carcinomas than in benign endometrial masses. In contrast, our study showed that fibrous cores and intratumoral cystic changes were seen more frequently in endometrial polyps than in endometrial cancer, in accord with Grasel et al.'s study [21]. Pedunculated lesions are usually considered benign. In the present study, only four benign lesions of the endometrium (4/35) were characterized as pedunculated. Hence, morphological features on T2WI might not be helpful in differentiating these lesions.

DWI is a functional imaging method based on the quantification of increased or restricted microscopic diffusion motions of tissue water molecules [14]. Increased tumor cellularity, which can restrict water diffusion, therefore decreases ADC values. Malignant lesions frequently have greater cellularity than benign lesions. Thus, ADC values can contribute to distinguishing between benign and malignant lesions [22-27]. Various

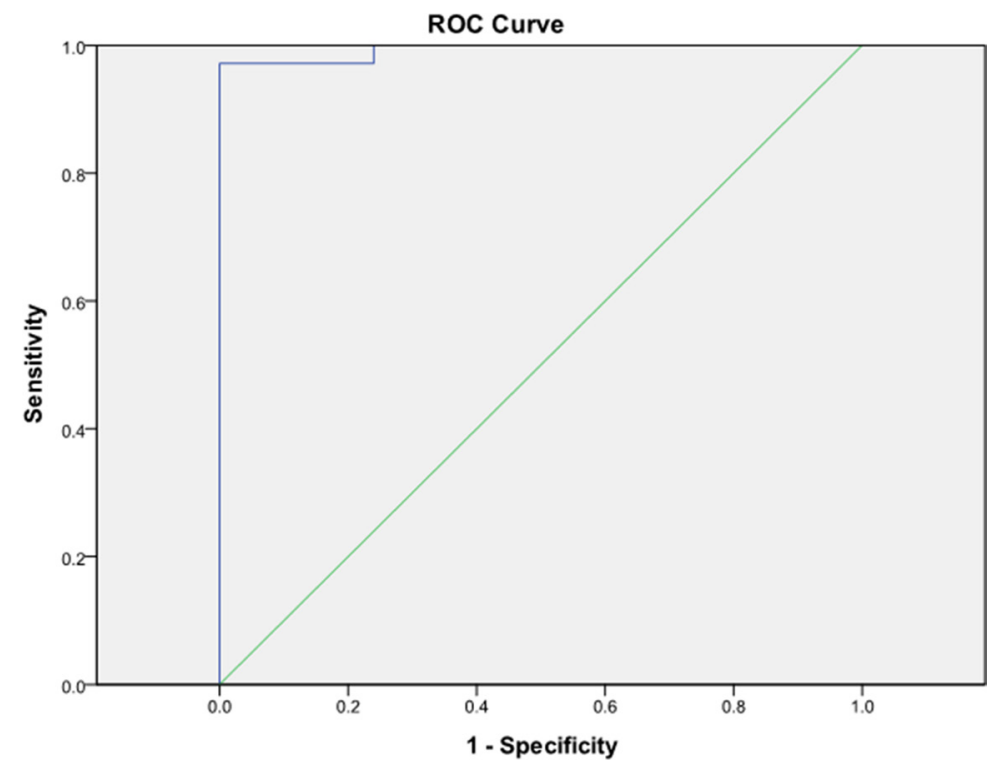

Figure 3: Receiver operating characteristic curve analysis showed an area under the curve for ADC values of 0.993 (95\% confidence interval 0-1). Cutoff value differentiating stage I endometrial carcinoma from benign lesions was $0.98 \times 10^{-3} \mathrm{~mm}^{2} / \mathrm{s}$. 
cutoff ADC values have been used to differentiate malignant from benign uterine lesions $[14,15,17,19,20]$. These cutoff ADC values ranged from $0.908 \times 10^{-3} \mathrm{~mm}^{2} / \mathrm{s}$ to $1.28 \times 10^{-3} \mathrm{~mm}^{2} / \mathrm{s}$. In this study, by applying the cutoff ADC value of $0.98 \times 10^{-3} \mathrm{~mm}^{2} / \mathrm{s}$, we achieved the greatest accuracy $(98.3 \%)$.

There are some major differences between previous studies and the present one. First, the previously published cutoff values were obtained at $1.5 \mathrm{~T}$ MRI in four studies $[14,15,19,20]$, and Takeuchi et al. [17] used both 1.5T and $3 \mathrm{~T}$ systems. In this study, we performed 3.0T MRI in all patients to enhance the signal-to-noise ratio. A phantom study [28] confirmed that ADC values vary at 1.5T and 3T. Second, using different $b$ values could affect the calculation of ADC values, causing variable results. Thus, it is difficult to establish a universal threshold for the ADC value. Third, because benign endometrial lesions and stage I endometrial carcinomas are similar, accurate diagnosis is difficult. Hence, all endometrial malignant lesions were known to be stage I endometrial carcinomas during the first stage of of this study. In contrast, previous studies included all endometrial carcinoma stages or carcinosarcomas. Lastly, the ADC value may relate to individual changes, such as age and body temperature, causing interpatient variation $[29,30]$.

In this study, we found that the ADC value for endometrial polyps was significantly higher than that for submucosal leiomyomas. Leiomyomas are composed of smooth muscle cells and collagen, which causes lower ADC values [31]. They are also associated with degeneration (e.g., hyalinization, edema), which occurs in more than $50 \%$ of leiomyomas [32]. Hyalinization leads to accumulation of homogeneous eosinophilic bands in the extracellular space, narrowing this space and thus decreasing ADC values [33]. In contrast, edema leads to increased ADC values because of widening of the extracellular space. The ADC value for leiomyoma is related to these histological findings and is thus complicated. Endometrial polyps contain endometrial glands and stroma or smooth muscle tissue. Cystic glandular hyperplasia generally develops in polyps. These changes can decrease cellularity and increase movement of water molecules, thus promoting increased ADC values $[14,34]$. Further studies in larger samples are needed to investigate a cutoff ADC value for the differential diagnosis of endometrial polyps and submucosal leiomyomas.

The cutoff ADC value obtained during the first stage of the study was prospectively applied in the second stage to validate its effectiveness. The high kappa value for agreement regarding measured ADC values between two radiologists indicates that this $\mathrm{ADC}$ value could be used reliably to assess endometrial lesions. The sensitivity, specificity, and accuracy of differentiating malignant endometrial lesions from benign lesions were high. These results indicated that the ADC value acquired using DWI could be a potential quantitative and qualitative biomarker to differentiate benign and malignant endometrial lesions. ADC values, however, might not be measured accurately in some small endometrial masses. In this situation, DWI could enhance its diagnostic capability if it is combined with dynamic contrast enhancement.

Our study has some limitations. First, endometrial carcinomas, polyps, and submucosal myomas $<10 \mathrm{~mm}$ or thick endometrium were not included in this study to ensure relatively reliable results. Second, the sample size for the second (prospective) stage of the study was relatively small to validate the cutoff $\mathrm{ADC}$ value obtained from the first (retrospective) stage of the study. Nevertheless, a PubMed search revealed no other publications that prospectively validated the use of ADC as a biomarker in the endometrial cavity.

The ADC values for stage I endometrial carcinomas were significantly lower than those for benign lesions, or "mimickers." The ADC value obtained with DWI could be a potential biomarker when used as a quantitative and qualitative tool for differentiating between early-stage endometrial carcinomas and their benign mimickers.

\section{MATERIALS AND METHODS}

The study was designed into two stages. The first stage, a retrospective study, was performed to evaluate the diagnostic value of ADC and obtained a theoretical ADC cutoff value for differentiating benign and malignant endometrial lesions. The second stage, a prospective study, was performed to validate the ADC cutoff value obtained for differentiating between benign and malignant endometrial lesions.

\section{Subjects}

Our institutional review board granted a waiver for consent because of the anonymous nature of this study. A total of 131 consecutive patients were identified. During the first stage, we excluded 6 patients with stage II-IV endometrial carcinomas and 5 patients whose imaging was of such poor quality we were unable to make a diagnosis. We excluded 10 patients due to endometrial hyperplasia with no mass in the endometrial cavity and 5 patients whose endometrial lesions were $<1.0 \mathrm{~cm}$ diameter (2 endometrial hyperplasia, 3 stage Ia endometrial carcinoma). During the second (prospective) stage of the study, we excluded 4 patients because their clinically suspicious endometrial masses were simply thick endometrium as shown on MRI, 5 patients whose endometrial lesions were $<1.0 \mathrm{~cm}$ diameter, and 10 patients whose lesions were not histologically confirmed. Finally, 86 patients (ages 30-75 years, mean 60 years) with International Federation of Gynecology and Obstetrics (FIGO) stage I endometrial carcinomas, submucosal leiomyomas, and polyps were enrolled in this 
study. All tumors were later confirmed histopathologically. Hysteroscopic polypectomy or myomectomy was performed for benign lesions and hysterectomy for malignant lesions. All patients underwent conventional MRI and DWI with $b$ values of $0 \mathrm{~s} / \mathrm{mm}^{2}$ and $1000 \mathrm{~s} / \mathrm{mm}^{2}$, respectively, before surgery. These lesions were assessed by two blinded experienced radiologists in gynecological imaging.

\section{Imaging protocol}

MRI was performed by a $3 \mathrm{~T}$ scanner (HDXT; General Electric Medical Systems, Milwaukee, WI, USA) with an 8-channel torso phased-array coil. All patients were examined with T1-weighted imaging (T1WI), T2weighted imaging (T2WI), and DWI. Parameters were as follows: (1) T1WI in axial oblique (to the corpus) planes: repetition time (TR)/time to echo (TE) 500-600/7-10 ms, field of view (FOV) $380 \times 380 \mathrm{~mm}$, section thickness $5.0 \mathrm{~mm}$, intersection gap $3 \mathrm{~mm}$; (2) short time inversion recovery T2WI in axial, sagittal, and coronal planes: TR/ TE 2900-3000/70-80 ms, FOV 280-380 × $380 \mathrm{~mm}$, section thickness $5 \mathrm{~mm}$, intersection gap 3.0-5.0 $\mathrm{mm}$. (3) Axial DWI of the pelvis: TR/TE 5000/66 ms, FOV $380 \times 380 \mathrm{~mm}$, matrix $96 \times 130$, section thickness $5 \mathrm{~mm}$, $\mathrm{b}=0$ and $1000 \mathrm{~s} / \mathrm{mm}^{2}$, respectively.

\section{Imaging analysis}

MRI images were retrospectively and blindly evaluated by two radiologists during the first stage of the study, with discrepancies resolved by a consensus discussion. On DWI, high-, iso-, or low-signal lesions, compared with the normal endometrium signals, were identified. Myometrial invasion was diagnosed when the endometrial-myometrial junction was disrupted. We obtained a cutoff ADC value for differentiating malignant and benign lesions from ROC curve. Other cutoff ADC values were obtained from published studies $[14,15,17,19,20]$, which along with our ADC, were used to calculate corresponding the sensitivity, specificity, and accuracy. During the second stage of the study, the cutoff ADC value obtained during the first stage was prospectively applied to the lesions to predict the diagnosis of either endometrial malignancy or a benign lesion.

\section{Imaging post-processing}

ADC maps were generated from a built-in software package (GE Func Tool Version 4.5) using $\mathrm{b}=0$ and $\mathrm{b}=$ $1000 \mathrm{~s} / \mathrm{mm}^{2}$ images. For each patient, a circular region of interest (ROI) $\left(55 \mathrm{~mm}^{2}\right)$ was created on central slices that contained the largest portion of the tumor. Each ROI was positioned on the solid area of the lesion while excluding any cystic and/or necrotic area and any endometrial tissue (Figure 4). The ADC values were then calculated automatically. The ROI was measured three times and the mean value recorded.

\section{Statistical analysis}

The Kolmogorov-Smirnov test was used to determine the normality of the data distributions of the ADC values for both stages of the study. For the first stage, Student's $t$-test was applied to compare the ADC values of malignant and benign tumors and between endometrial polyps and submucosal leiomyomas. The ROC curve was used to obtain a cutoff ADC value for differentiating stage I endometrial carcinomas from benign lesions. The sensitivity, specificity, and accuracy were also calculated.
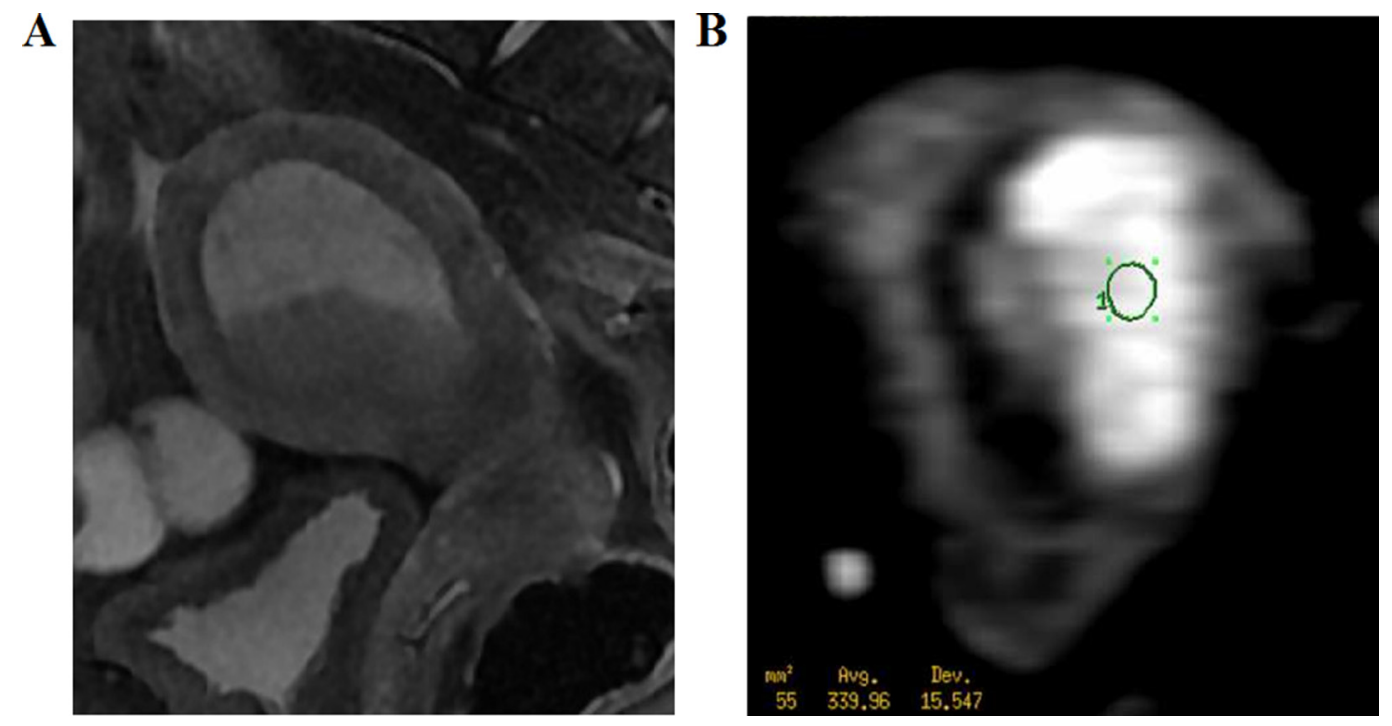

Figure 4: A 62-year-old woman with stage I endometrial carcinoma. (A) Sagittal T2WI shows the carcinoma with slightly high intensity (arrow). (B) Axial DWI shows a carcinoma with hyperintensity. The region of interest $\left(55 \mathrm{~mm}^{2}\right)$ was placed at the center of the lesion. 
For the second stage of the study, the cutoff ADC value attained during the first stage was prospectively applied to lesions to calculate its sensitivity, specificity, and accuracy to validate its diagnostic performance. The kappa test was used to assess the agreement between the two radiologists. SPSS software 18.0 (SPSS, Chicago, IL, USA) was used for the statistical analyses. $P<0.05$ was considered to indicate statistical significance.

\section{Abbreviations}

ADC: apparent diffusion coefficient; AUC: area under the curve; MRI: magnetic resonance imaging; DWI: diffusion-weighted imaging; TR/TE: repetition time/time to echo; FOV: field of view; ROC: receiver operating characteristic; ROI: region of interest.

\section{Authors' contributions}

The paper was designed and described by Jinhong Wang and Zhihan Yan. It was written and analyzed by Xue Wang. Yu Zhao, Yumin Hu, Yongjin Zhou, Meimei $\mathrm{Du}$ and Anna Guo diagnosed the cases in this study and established the inclusion and exclusion criteria for the patients. Kun Liu, Guanghui Bai and Lezhen Jiang analyzed the data obtained and performed the statistical analyses.

\section{CONFLICTS OF INTEREST}

The authors declare no conflicts of interest.

\section{FUNDING}

This study was funded by the National Natural Science Foundation of China (No. 81400863), Zhejiang Provincial Natural Science Foundation (Nos. LY15H180010, LY16H180009), and Wenzhou Science and Technology Bureau in China (No. Y20160479).

\section{REFERENCES}

1. van Dongen $\mathrm{H}$, de Kroon $\mathrm{CD}$, Jacobi CE, Trimbos JB, Jansen FW. Diagnostic hysterescopy in abnormal uterine bleeding: a systematic review and meta-analysis. BJOG. 2007; 114:664-675.

2. Hase S, Mitsumori A, Inai R, Takemoto M, Matsubara S, Akamatsu N, Fujisawa M, Joja I, Sato S, Kanazawa S. Endometrial polyps: MR imaging features. Acta Med Okayama. 2012; 66:475-85.

3. Takeuchi M, Matsuzaki K, Uehara H, Yoshida S, Nishitani H, Shimazu H. Pathologies of the uterine endometrial cavity: usual and unusual manifestations and pitfalls on magnetic resonance imaging. Eur Radiol. 2005; 15:2244-2255.
4. Shen SH, Chiou YY, Wang JH, Yen MS, Lee RC, Lai CR, Chang CY. Diffusion-weighted single-shot echoplanar imaging with parallel technique in assessment of endometrial cancer. AJR Am J Roentgenol. 2008; 190:481-488.

5. Wang J, Yu T, Bai R, Sun H, Zhao X, Li Y. The Value of the apparent diffusion coefficient in differentiating stage IA endometrial carcinoma from normal endometrium and benign diseases of the endometrium: initial study at 3-T magnetic resonance scanner. J Comput Assist Tomoqr. 2010; 34:332-337.

6. Ohguri T, Aoki T, Watanabe H, Nakamura K, Nakata H, Matsuura Y, Kashimura M. MRII findings including gadolinium-enhanced dynamic studies of malignant, mixed mesodermal tumors of the uterus: differentiation from endometrial carcinomas. Eur Radiol. 2002; 12:2737-2742.

7. Park BK, Kim B, Park JM, Ryu JA, Kim MS, Bae DS, Ahn GH. Differentiation of the various lesions causing an abnormality of the endometrial cavity using MRI imaging: emphasis on enhancement. Patterns on dynamic studies and late contrast-enhanced T1-weighted images. Eur Radiol. 2006; 16:1591-1598.

8. Nakai G, Matsuki M, Inada Y, Tatsugami F, Tanikake M, Narabayashi I, Yamada T. Detection and evaluation of pelvic lymph nodes in patients with gynecologic malignancies using body diffusion-weighted magnetic resonance imaging. J Comput Assist Tomogr. 2008; 32:764-768.

9. Granata V, Fusco R, Catalano O, Guarino B, Granata F, Tatangelo F, Avallone A, Piccirillo M, Palaia R, Izzo F, Petrillo A. Intravoxel incoherent motion (IVIM) in diffusion-weighted imaging (DWI) for Hepatocellular carcinoma: correlation with histologic grade. Oncotarget. 2016; 7:79357-79364. doi: 10.18632/oncotarget.12689.

10. Nakamura Y, Bernardo M, Nagaya T, Sato K, Harada T, Choyke PL, Kobayashi H. MR imaging biomarkers for evaluating therapeutic effects shortly after near infrared photoimmunotherapy. Oncotarget. 2016; 7:17254-17264. doi: 10.18632/oncotarget.7357.

11. Naganawa S, Sato C, Kumada H, Ishigaki T, Miura S, Takizawa O. Apparent diffusion coefficient in cervical cancer of the uterus: comparison with the normal uterine cervix. Eur Radiol. 2005; 15:71-78.

12. Sato C, Naganawa S, Nakamura T, Kumada H, Miura S, Takizawa O, Ishigaki T. Differentiation of noncancerous tissue and cancer lesions by apparent diffusion coefficient values in transition and peripheral zones of the prostate. $\mathrm{J}$ Magn Reson Imaging. 2005; 21:258-262.

13. Uhl M, Altehoefer C, Kontny U, Il'yasov K, Büchert M, Langer M. MRII-diffusion imaging of neuroblastomas: first results and correlation to histology. Eur Radiol. 2002; 12:2335-2338.

14. Fujii S, Matsusue E, Kigawa J, Sato S, Kanasaki Y, Nakanishi J, Sugihara S, Kaminou T, Terakawa N, Ogawa T. Diagnostic accuracy of the apparent diffusion coefficient in differentiating benign from malignant uterine endometrial cavity lesions: initial results. Eur Radiol. 2008; 18:384-389. 
15. Kilickesmez O, Bayramoglu S, Inci E, Cimilli T, Kayhan A. Quantitative diffusion-weighted magnetic resonance imaging of normal and diseased uterine zones. Acta Radiol. 2009; 50:340-347.

16. Inada Y, Matsuki M, Nakai G, Tatsugami F, Tanikake M, Narabayashi I, Yamada T, Tsuji M. Body diffusion-weighted MRI imaging of uterine endometrial cancer: is it helpful in the detection of cancer in nonenhanced MRI imaging? Eur J Radiol. 2009; 70:122-127.

17. Takeuchi M, Matsuzaki K, Nishitani H. Diffusion weighted magnetic resonance imaging of endometrial cancer: differentiation from benign endometrial lesions and preoperative assessment of myometrial invasion. Acta Radiol. 2009; 50:947-953.

18. Seo JM, Kim CK, Choi D, Kwan Park B. Endometrial cancer: utility of diffusion weighted magneticresonance imaging with background bodysignal suppression at 3T. J Mgn Reson Imaging. 2013; 37:1151-1159.

19. Bharwani N, Miquel ME, Sahdev A, Narayanan $P$, Malietzis G, Reznek RH, Rockall AG. Diffusionweighted Imaging in the assessment of tumour grade in endometrial cancer. Br J Radiol. 2011; 84:997-1004.

20. Karakas O, Karakas E, Dogan F, Kilicaslan N, Camuzcuoglu A, Incebiyik A, Camuzcuoglu H. Diffusion-weighted MRII in the differential diagnosis of uterine endometrial cavity tumors. Wien Klin Wochenschr. 2015; 127:266-273.

21. Grasel RP, Outwater EK, Siegelman ES, Capuzzi D, Parker L, Hussain SM. Endomeuial polyps: MRI imaging features and distinction from endometrial carcinonm. Radiology. 2000; 214:47-52.

22. Mukherji SK, Chenevert TL, Castillo M. Diffusionweighted magnetic resonance imaging. J Neuroophthalmol. 2002; 22:118-22.

23. Sugahara T, Korogi Y, Kochi M, Ikushima I, Shigematu Y, Hirai T, Okuda T, Liang L, Ge Y, Komohara Y, Ushio Y, Takahashi M. Usefulness of diffusion-weighted MRII with echo-planar technique in the evaluation of cellularity in gliomas. J Magn Reson Imaging. 1999; 9:53-60.

24. Gauvain KM, McKinstry RC, Mukherjee P, Perry A, Neil JJ, Kaufman BA, Hayashi RJ. Evaluating pediatric brain tumor cellularity with diffusion-tensor imaging. AJR Am J Roentgenol. 2001; 177:449-454.

25. Guo AC, Cummings TJ, Dash RC, Provenzale JM. Lymphomas and high-grade astrocytomas: comparison of water diffusibility and histologic characteristics. Radiology. 2002; 224:177-183.
26. Rumboldt Z, Camacho DL, Lake D, Welsh CT, Castillo M. Apparent diffusion coefficients for differentiation of cerebellar tumors in children. AJNR Am J Neuroradiol. 2006; 27:1362-1369.

27. Hayashida Y, Hirai T, Morishita S, Kitajima M, Murakami R, Korogi Y, Makino K, Nakamura H, Ikushima I, Yamura M, Kochi M, Kuratsu JI, Yamashita Y. Diffusion-weighted imaging of metastatic brain tumors: comparison with histologic type and tumor cellularity. AJNR Am J Neuroradiol. 2006; 27:1419-1425.

28. Chenevert TL, Galbán CJ, Ivancevic MK, Rohrer SE, Londy FJ, Kwee TC, Meyer CR, Johnson TD, Rehemtulla A, Ross BD. Diffusion coefficient measurement using a temperaturecontrolled fluid for quality control in multicenter studies. J Magn Reson Imaging. 2011; 34:983-987.

29. Jacobs MA, Ouwerkerk R, Petrowski K, Macura KJ. Diffusion weighted imaging with apparent diffusion coefficient mapping and spectroscopy in prostate cancer. Top Magn Reson Imaging. 2008; 19:261-272.

30. Tamada T, Sone T, Toshimitsu S, Imai S, Jo Y, Yoshida K, Yamamoto A, Yamashita T, Egashira N, Nagai K, Fukunaga $\mathrm{M}$. Age-related and zonal anatomical changes of apparent diffusion coefficient values in normal human prostatic tissues. J Magn Reson Imaging. 2008; 27:552-556.

31. Liapi E, Kamel IR, Bluemke DA, Jacobs MA, Kim HS. Assessment of response of uterine fibroids and myometrium to embolization using diffusion-weighted echoplanar MRI imaging. J Comput Assist Tomogr. 2005; 29:83-86.

32. Ueda H, Togashi K, Konishi I, Kataoka ML, Koyama T, Fujiwara T, Kobayashi H, Fujii S, Konishi J. Unusual appearances of uterine leiomyomas: MRI imaging findings and their histo-pathologic backgrounds. Radiographics. 1999; 19:S131-S145.

33. Shimada K, Ohashi I, Kasahara I, Watanabe H, Ohta S, Miyasaka N, Itoh E, Shibuya H. Differentiation between completely hyalinized uterine leiomyomas and ordinary leiomyomas: three-phase dynamic magnetic resonance imaging (MRII) vs. diffusion-weighted MRII with very small b-factors. J Magn Reson Imaging. 2004; 20:97-104.

34. Vermoolen MA, Kwee TC, Nievelstein RA. Apparent diffusion coefficient measurements in the differentiation between benign and malignant lesions: a systematic review. Insights into imaging. 2012; 3:395-409. 